\title{
Downregulation of miR-486-5p alleviates LPS-induced inflammatory injury, oxidative stress and apoptosis in Chondrogenic cell ATDC5 by targeting NRF1
}

\author{
QING CHANG, MINGLIANG JI, CHAO LI and RUI GENG \\ Department of Orthopaedic Surgery, Zhongda Hospital, School of Medicine, \\ Southeast University, Nanjing, Jiangsu 210009, P.R. China
}

Received September 9, 2019; Accepted March 2, 2020

DOI: $10.3892 / \mathrm{mmr} .2020 .11289$

\begin{abstract}
Osteoarthritis (OA) is one of the most common degenerative disease in elderly patients worldwide. Numerous microRNAs (miRs) have been reported to serve an important role in the regulation of gene expression in the occurrence and development of OA. The present study aimed to explore the effect of miR-486-5p on lipopolysaccharide (LPS)-induced cell damage in chondrocytes, as well as the underlying mechanism. The ATDC5 cell line was treated with increasing concentrations of LPS $(0,1,2,4$ and $8 \mu \mathrm{g} / \mathrm{ml})$ for $6 \mathrm{~h}$. The binding site of $\mathrm{miR}-486-5 \mathrm{p}$ on nuclear factor erythroid 2 like 1 (NRF1) was predicted using the miRDB database and was validated using the luciferase reporter assay. A CCK-8 assay and flow cytometry analysis were conducted to determine cell viability and apoptosis, respectively. The level of inflammatory cytokines and oxidative stress-associated factors were detected using corresponding test kits. Furthermore, the expression of associated genes were detected using reverse transcription-quantitative PCR and western blotting. LPS significantly decreased cell proliferation, induced cell apoptosis and aggravated the inflammatory response and oxidative stress. Furthermore, miR-486-5p and NRF1 were significantly upregulated and downregulated, respectively, in LPS-induced ATDC5 cells. miR-486-5p was identified to directly target and regulate the expression of NRF1. Inhibition of miR-486-5p significantly improved cell proliferation, decreased apoptosis, attenuated the production of inflammatory cytokines, regulated the level of reactive oxygen species, malondialdehyde, superoxide dismutase and lactate dehydrogenase, and improved the activity of antioxidant enzyme. Furthermore, the effect of
\end{abstract}

Correspondence to: Dr Qing Chang, Department of Orthopaedic Surgery, Zhongda Hospital, School of Medicine, Southeast University, 87 Ding Jiaqiao Road, Nanjing, Jiangsu 210009, P.R. China E-mail: changqq777@163.com

Key words: microRNA-486-5p, inflammatory injury, oxidative stress, apoptosis, nuclear factor ery throid 2 like 1, osteoarthritis
miR-486-5p on LPS-induced cell damage was diminished following the downregulation of NRF1. To conclude, inhibition of miR-486-5p alleviated LPS-induced cell damage, including inflammatory injury, oxidative stress and apoptosis, in ATDC5 cells by targeting NRF1. Therefore, NRF1 may serve as a novel therapeutic target for OA.

\section{Introduction}

Osteoarthritis (OA) is a common chronic arthritis affecting cartilaginous tissues that generally occurs in middle-aged and elderly patients and affects $\sim 40 \%$ of individuals $>70$ years of age (1). The common clinical manifestations of OA are pain, and joint dysfunction and deformity, and OA is regarded as one of the primary causes of disability, representing a major healthcare issue for both governments and researchers (2). Previous studies have demonstrated that numerous factors contribute to the occurrence of OA, including obesity, aging, inflammation and physical strain (3-5). Due to the high prevalence of obesity and age-associated diseases, OA is associated with significant morbidity worldwide (6). Therefore, an increased understanding of the pathological mechanisms underlying OA, particularly degeneration of articular cartilage, may prevent the progression of the disease.

Nuclear factor erythroid 2 like 1 (NRF1) is a basic leucine zipper protein that belongs to the cap-N-collar transcriptional factor family (7). NRF1 is produced by osteoblasts, and is involved in regulating osterix expression, osteoblast differentiation and bone formation (8). NRF1 was shown to serve important roles in rheumatoid arthritis (9). Additionally, NRF1 serves an important role in the regulation of antioxidant enzymes during inflammation-induced oxidative stress in osteoblastic cells (10). The aforementioned data suggest that NRF1 is closely associated with the development of arthritis, partly by regulating cellular differentiation, inflammation and oxidative stress. However, to the best of our knowledge, the function of NRF1 in the progression of OA has not been previously investigated.

MicroRNAs (miRs/miRNAs) are a group of short, non-coding RNAs that serve an important role in regulating gene expression. Furthermore, miRNAs have been implicated in the progression of various diseases, including OA (11). 
Kong et al (12), revealed that miR-486-5p was upregulated in patients with knee OA, and was positively correlated with the severity of the disease. Additionally, miR-486-5p was reported to target SMAD family member 2 , and negatively regulate the expression of collagen type II and aggrecan in chondrocytes, aggravating the progression of OA (13). However, to the best of our knowledge, the role of miR-486-5p in OA has only been investigated in the aforementioned two studies, and whether miR-486-5p could regulates NRF1 remains unknown. Therefore, the mechanisms underlying miR-486-5p and OA require further investigation.

The present study aimed to explore the roles of miR-486-5p and NRF1 in OA, and to explore the underlying mechanism of molecular action, which may provide a novel treatment strategy for OA.

\section{Materials and methods}

Cell culture and treatment. The murine chondrogenic ATDC5 cell line was obtained from the American Type Culture Collection. The cells were cultured in DMEM: Ham's F-12 (1:1; Sigma-Aldrich; Merck KGaA) supplemented with $2 \mathrm{mM}$ glutamine (Sigma-Aldrich; Merck KGaA) and 10\% FBS (Invitrogen; Thermo Fisher Scientific, Inc.) and maintained at $37^{\circ} \mathrm{C}$ in a humidified incubator containing $5 \% \mathrm{CO}_{2}$. ATDC5 cells were then treated with lipopolysaccharide (LPS; Sigma-Aldrich; Merck KGaA) at different concentrations $(0,1,2,4$ and $8 \mu \mathrm{g} / \mathrm{ml})$ at $37^{\circ} \mathrm{C}$ for $6 \mathrm{~h}$ to induce inflammatory injury.

Cell Counting Kit-8 (CCK-8) assay. The proliferation of ATDC5 cells was determined using a CCK-8 assay kit (Dojindo Molecular Technologies, Inc.), according to the manufacturer's protocol. Cells $\left(5 \times 10^{3}\right.$ cells/well) were seeded into a 96-well plate and cultured for $24 \mathrm{~h}$. After treatment with LPS for $6 \mathrm{~h}$, $20 \mu \mathrm{l} \mathrm{CCK}-8$ solution was added into each well and incubated for another $4 \mathrm{~h}$ at $37^{\circ} \mathrm{C}$. The absorbance was subsequently detected at a wavelength of $450 \mathrm{~nm}$ using a microplate reader.

Western blot analysis. ATDC5 cells were lysed with cold RIPA lysis buffer (Beyotime Institute of Biotechnology) supplemented with protease inhibitor. The concentration of protein was measured using the BCA protein assay (Pierce; Thermo Fisher Scientific, Inc.). The proteins (20 $\mu \mathrm{g} /$ lane) were separated using $10 \%$ SDS-PAGE, and transferred onto PVDF membranes. Membranes were blocked with 5\% skimmed milk at room temperature for $1 \mathrm{~h}$ and incubated with the primary antibodies with a dilution of 1:1,000 overnight at $4^{\circ} \mathrm{C}$. The antibodies against NRF1 (cat. no. ab137572), Bax (cat. no. ab32503), Bcl-2 (cat. no. ab59348), cleaved caspase-3 (cat. no. ab49822), caspase-3 (cat. no. ab32499), superoxide dismutase 1 (SOD1; cat. no. ab13498), heme oxygenase-1 (HO-1; cat. no. ab13243), NAD(P)H quinone dehydrogenase 1 (NQO-1; cat. no. ab34173) and GAPDH (cat. no. ab9485) were obtained from Abcam. Following incubation with the primary antibodies, the membranes were incubated with a horseradish peroxidase-conjugated secondary antibody (1:5,000; cat. no. 7074; Cell Signaling Technology, Inc.) for $2 \mathrm{~h}$ at room temperature. Bands were visualized using an enhanced chemiluminescence kit (SuperSignal ${ }^{\mathrm{TM}}$ West Pico PLUS chemiluminescent substrate; Thermo Fisher Scientific,
Inc.). The densitometric analysis was performed using ImageJ software v1.4 (National Institutes of Health).

Reverse transcription-quantitative $(R T-q) P C R$. Total RNA was extracted from ATDC5 cells using TRIzol reagent (Invitrogen; Thermo Fisher Scientific, Inc.). To detect the mRNA levels of miR-486-5p, the first strand of cDNA was synthesized using the PrimeScript RT reagent kit (Takara Biotechnology Co., Ltd.), according to the manufacturer's protocol, and the following temperature conditions: Initial incubation at $37^{\circ} \mathrm{C}$ for $15 \mathrm{~min}$, followed by an incubation at $85^{\circ} \mathrm{C}$ for $5 \mathrm{sec}$. RT-qPCR was performed using a SYBR-Green Prime Script RT-PCR kit (Takara Biotechnology Co., Ltd.), according to the manufacturer's instructions. The thermocycler conditions for RT-qPCR were as follows: $95^{\circ} \mathrm{C}$ for $5 \mathrm{~min}$, followed by 40 cycles of denaturation at $95^{\circ} \mathrm{C}$ for $15 \mathrm{sec}$ and annealing/extension at $60^{\circ} \mathrm{C}$ for $30 \mathrm{sec}$. The primer sequences were as follows: miR-486-5p forward, 5'-CTCGCTTCGGCAGCACA-3'; miR-486-5p reverse 5'-ACGCTTCACGAATTTGCGT-3'; NRF1 forward, 5'- GGAGAGCTTCCCTGCACAGT-3'; NRF1 reverse, 5'- TTACTTCCATAGCCTGCATTTCC-3'; $\beta$-actin forward, 5'-CCGCGAGCACAGCTTCTT-3'; $\beta$-actin reverse, 5'-CCCACGATGGAGGGGAATAC-3'; U6 forward, 5'-CTCGCTTCGGCAGCACA-3'; and U6 reverse, 5'-AACGCTTCACGAATTTGCGT-3'. Data were collected and analyzed using $2^{-\Delta \Delta \mathrm{Cq}}$ method (14) and normalized to the internal control U6 and $\beta$-actin.

Cell transfection. ATDC5 cells were transfected with the miR-486-5p inhibitor (5'-CUCGGGGCAGCUCAGUAC AGGA-3'; $150 \mathrm{nM}$; Qiagen $\mathrm{GmbH}$ ) and inhibitor control (miR-NC; 5'-UUCUCCGAACGUGUCACGUTT-3'; 150 nM; Qiagen $\mathrm{GmbH}$ ), or transfected with short hairpin RNA (shRNA)1 or shRNA2 targeting NRF1 (100 nM) and its scramble control shRNA (shRNA-NC; empty vector), which were cloned into pSilencer 3.1-H1 puro plasmids (Shanghai GenePharma Co. Ltd.), using Lipofectamine ${ }^{\circledR} 2000$ reagent (Thermo Fisher Scientific, Inc.), according to the manufacturer's protocol. The transfection efficiency was detected by RT-qPCR $48 \mathrm{~h}$ post-transfection.

Dual luciferase reporter assay. The binding site of miR-486-5p on NRF1 was predicted using the miRDB database (www. mirdb.org) and was validated using the luciferase reporter assay. PmirGLO-NRF1-WT or pmirGLO-NRF1-Mut reporter plasmids (Promega Corporation) were co-transfected with the miR-486-5p mimic or miR-NC into ATDC5 cells using Lipofectamine $^{\circledR} 2000$ reagent. The luciferase activity was measured using a Dual-Luciferase ${ }^{\circledR}$ Reporter assay system (Promega Corporation) $48 \mathrm{~h}$ post-transfection. The data were standardized to Renilla luciferase activity.

Inflammatory cytokines assay. The cell culture medium of ATDC5 cells was collected. The levels of tumor necrosis factor- $\alpha$ (TNF- $\alpha$ ), interleukin (IL)-1 $\beta$, IL-6, monocyte chemotactic protein 1 (MCP-1) and intercellular adhesion molecule 1 (ICAM-1) were determined using ELISA kits (cat. no. 210-TA-005 for TNF- $\alpha$; cat. no. 201-LB-005 for IL-1 $\beta$; cat. no. S6050 for IL-6; cat. no. SCP00 for MCP-1; cat. no. 796-IC-050 for ICAM-1; R\&D Systems, Inc.) according to the manufacturer's instructions. 

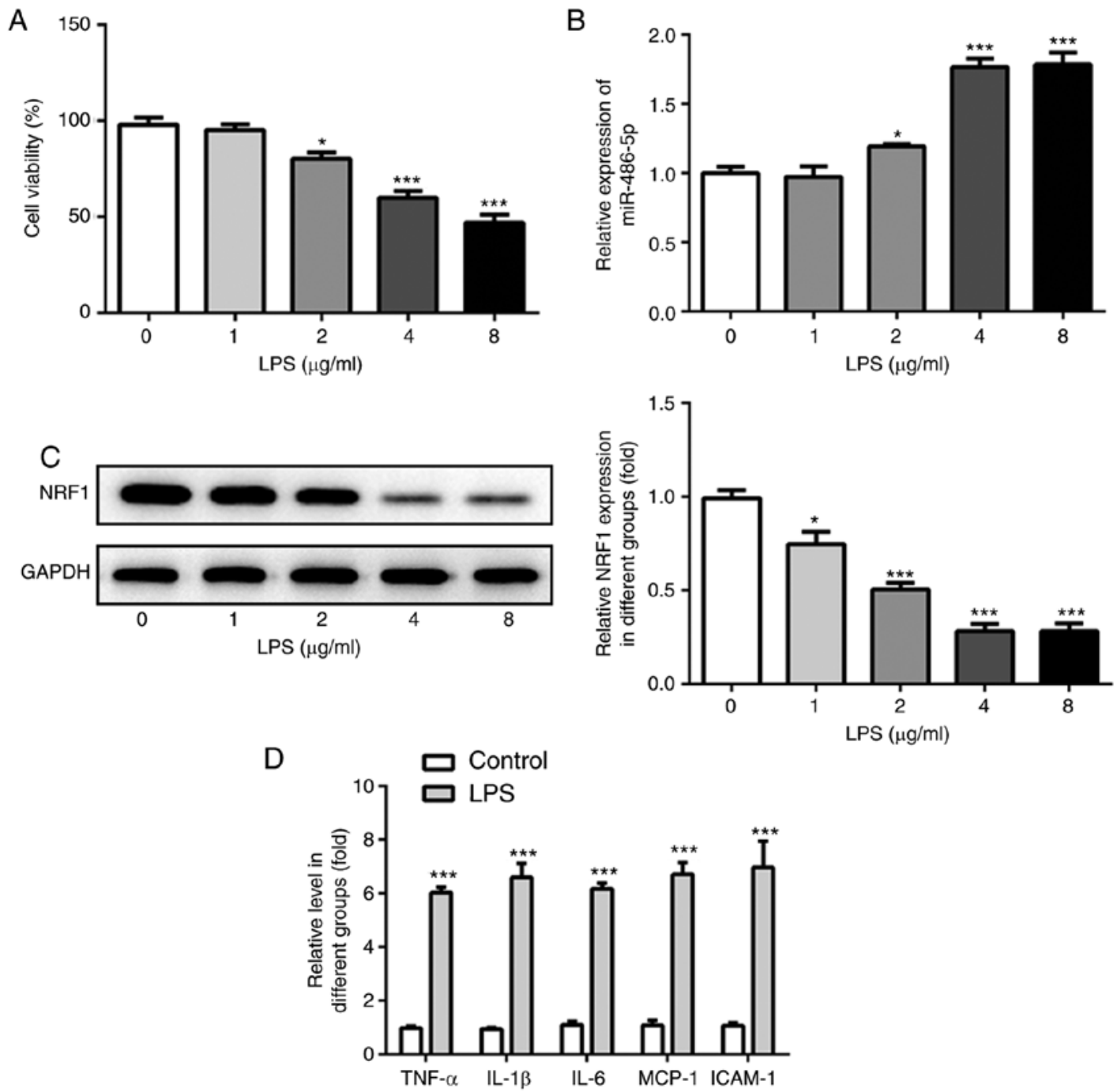

Figure 1. Expression of miR-486-5p and NRF1 in LPS-induced ATDC5 cells. Following induction of LPS (0, 1, 2, 4 and $8 \mu \mathrm{g} / \mathrm{ml})$ in ATDC cells, (A) cell viability was detected using a CCK-8 assay, (B) mRNA level of miR-486-5p was detected using reverse transcription-quantitative PCR, and (C) protein expression of NRF1 was detected using western blotting. ${ }^{*} \mathrm{P}<0.05$ and ${ }^{* * *} \mathrm{P}<0.001$ vs. 0 . (D) Levels of inflammatory cytokines, including TNF- $\alpha$, IL-1 $\beta$, IL-6, MCP-1 and ICAM-1 were detected by ELISA kits. ${ }^{* * *} \mathrm{P}<0.001$ vs. control. Data were generated from at least 3 experiments. miR, microRNA; LPS, lipopolysaccharide; NRF1, nuclear factor erythroid 2 like 1; TNF- $\alpha$, tumor necrosis factor $\alpha$; IL, interleukin; MCP-1, monocyte chemotactic protein 1; ICAM-1, intercellular adhesion molecule 1.

Oxidative stress factors assay. The cell culture medium of ATDC5 cells was collected. The levels of reactive oxygen species (ROS; cat. no. E004-1-1), malondialdehyde (MDA; cat. no. A003-4-1), SOD (cat. no. A001-3-2) and lactate dehydrogenase (LDH; A020-2-2) were determined using their corresponding assay test kits (Nanjing Jiancheng Bioengineering Institute), according to the manufacturers' instructions.

Flow cytometry. The apoptosis of ADTC5 cells was analyzed using an Annexin V-fluorescein isothiocyanate (FITC)/propidium iodide (PI) apoptosis detection kit (Sigma-Aldrich; Merck KGaA). Following treatment of LPS, the transfected ATDC5 cells were washed with PBS and incubated with Annexin V-FITC/PI $(50 \mu \mathrm{g} / \mathrm{ml})$ in the presence of RNase A (50 $\mu \mathrm{g} / \mathrm{ml}$; Sigma-Aldrich; Merck KGaA) at room temperature for $30 \mathrm{~min}$ in the dark. The apoptotic cells were detected by a FACScan flow cytometer (BD Biosciences) and analyzed using FlowJo software v7.6 (FlowJo LLC).
Statistical analysis. Statistical analyses were performed using SPSS software (v.17.0; SPSS, Inc.). Data are presented as the mean \pm SD of 3 independent experiments. Data were analyzed by the one-way ANOVA followed by the Tukey's post hoc test. $\mathrm{P}<0.05$ was considered to indicate a statistically significant different difference.

\section{Results}

Expression of miR-486-5p and NRF1 in LPS-induced ATDC5 cells. Following treatment of ATDC cells with LPS $(0,1,2$, 4 and $8 \mu \mathrm{g} / \mathrm{ml}$ ), the cell proliferation was detected using a CCK-8 kit, and the mRNA and protein expression levels of miR-486-5p and NRF1 were detected using RT-qPCR and western blotting, respectively. As shown in Fig. 1A, LPS treatment $(2,4$ and $8 \mu \mathrm{g} / \mathrm{ml})$ significantly decreased the proliferation in a concentration-dependent manner. Furthermore, the mRNA level of miR-486-5p was increased following LPS treatment (2, 4 and $8 \mu \mathrm{g} / \mathrm{ml}$; Fig. 1B). Protein expression of 

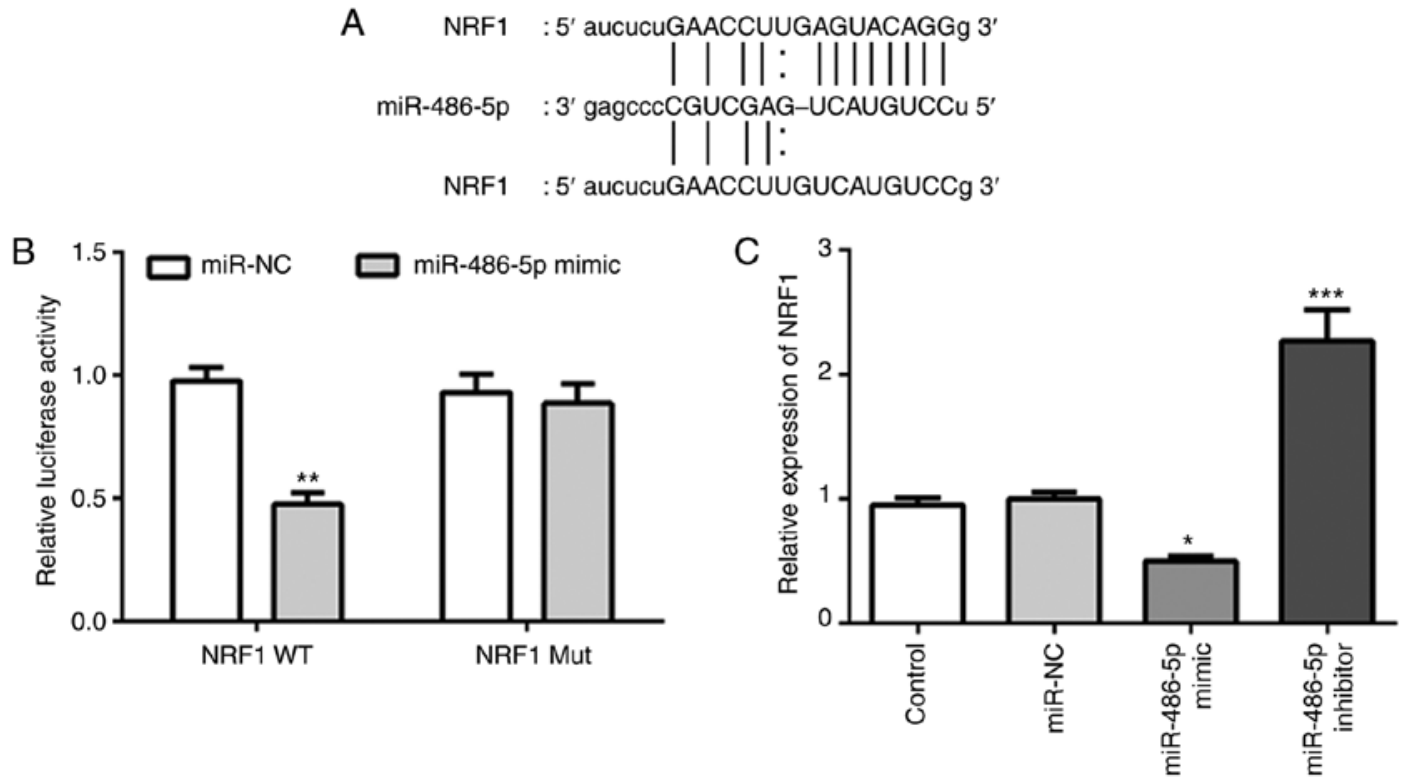

Figure 2. NRF1 is a target of miR-486-5p in ATDC cells. (A) The miRDB online database was used to identify the binding site of miR-486-5p and NRF1. (B) A luciferase reporter assay was performed to validate the interaction between miR-486-5p and NRF1.** $\mathrm{P}<0.01$ vs. miR-NC. (C) Expression of NRF1 was detected using reverse transcription-quantitative PCR following the transfection of miR-NC, miR-486-5p mimic and miR-486-5p inhibitor. ${ }^{*}<0.05$ and ${ }^{* * * *} \mathrm{P}<0.001$ vs. control. Data were generated from at least 3 experiments. miR, microRNA; NRF1, nuclear factor erythroid 2 like 1; NC, negative control; WT, wild type; Mut, mutant.

NRF1 was significantly decreased following LPS treatment, particularly at $4 \mu \mathrm{g} / \mathrm{ml}$ of LPS (Fig. 1C). Therefore, this concentration was chosen for subsequent experimentation. The results revealed that miR-486-5p was upregulated while NRF1 was downregulated in LPS-induced ATDC5 cells. To determine the LPS-induced inflammatory injury in ATDC5 cells, the levels of inflammatory cytokines including TNF- $\alpha$, IL-1 $\beta$, IL-6, MCP-1 and ICAM-1 were assayed by ELISA kits . Results in Fig. 1D indicated that LPS induced a significant increase in these inflammatory cytokines, revealing a marked inflammatory injury induced by LPS.

NRF1 is a target of miR-486-5p in ATDC cells. Analysis of the online databases of miRDB revealed that the 3'-UTR of NRF1 included a binding site for miR-486-5p (Fig. 2A). A luciferase reporter assay was subsequently performed to validate the interaction between miR-486-5p and NRF1 in ATDC cells. As shown in Fig. 2B, overexpression of miR-486-5p significantly suppressed the activity of the reporter gene, whereas the plasmid containing the mutated sequence did not affect the activity of the reporter gene in ADTC cells. Besides, overexpression of miR-486-5p decreased the expression of NRF1, while inhibition of miR-486-5p increased the expression of NRF1 (Fig. 2C). These results suggested that miR-486-5p could directly target NRF1 and regulate the expression of NRF1 in ATDC5 cells.

miR-486-5p regulates the LPS-induced inflammatory response in ATDC5 cells by targeting NRF1. The effect of miR-486-5p and NRF1 on LPS-induced ATDC5 cells was investigated. A miR-486-5p inhibitor or shRNA targeting NRF1 was transfected into cells. As shown in Fig. 3A and B, the increased mRNA level of miR-486-5p induced by LPS was significantly decreased following transfection with the
miR-486-5p inhibitor. Furthermore, the expression of NRF1 was significantly decreased when cells were transfected with shRNA1 or shRNA2 targeting NRF1. Due to a higher transfection efficacy, shRNA1-NRF1 was used for the further experimentation.

The change of inflammatory cytokines, directly reflecting the degree of inflammatory injury in LPS-induced ATDC5 cells, is presented in Fig. 3C-G. As observed from the results, LPS induction significantly increased the production of TNF- $\alpha$, IL-1 $\beta$, IL-6, MCP-1 and ICAM-1. As expected, the production of these inflammatory cytokines was decreased following transfection with the miR-486-5p inhibitor, and co-transfection with shRNA-NRF1 abrogated this effect. These results suggested that the inhibition of miR-486-5p may significantly alleviate LPS-induced inflammatory injury in ATDC 5 cells by regulating NRF1.

miR-486-5p regulates LPS-induced cell apoptosis by targeting NRF1. The effect of miR-486-5p on cell apoptosis in LPS-induced ATDC5 cells was investigated. Flow cytometry analysis revealed an increased apoptosis rate following LPS induction, whereas in the miR-486-5p inhibitor group the apoptotic rate of the cells was decreased compared with the LPS group (Fig. 4A). Additionally, the expression levels of apoptosis-associated proteins were investigated by western blotting. LPS induction significantly decreased the protein expression of $\mathrm{Bcl}-2$, and increased the protein expression of Bax and cleaved caspase-3 (Fig. 4B), indicating that LPS treatment resulted in ATDC cell apoptosis by regulating the expression of apoptosis-associated proteins. Furthermore, the expressions of Bax and cleaved caspase- 3 was decreased, and Bcl-2 was increased in the miR-486-5p inhibitor group, suggesting an inhibitory effect of miR-486-5p in LPS-induced ATDC cells. The inhibitory 

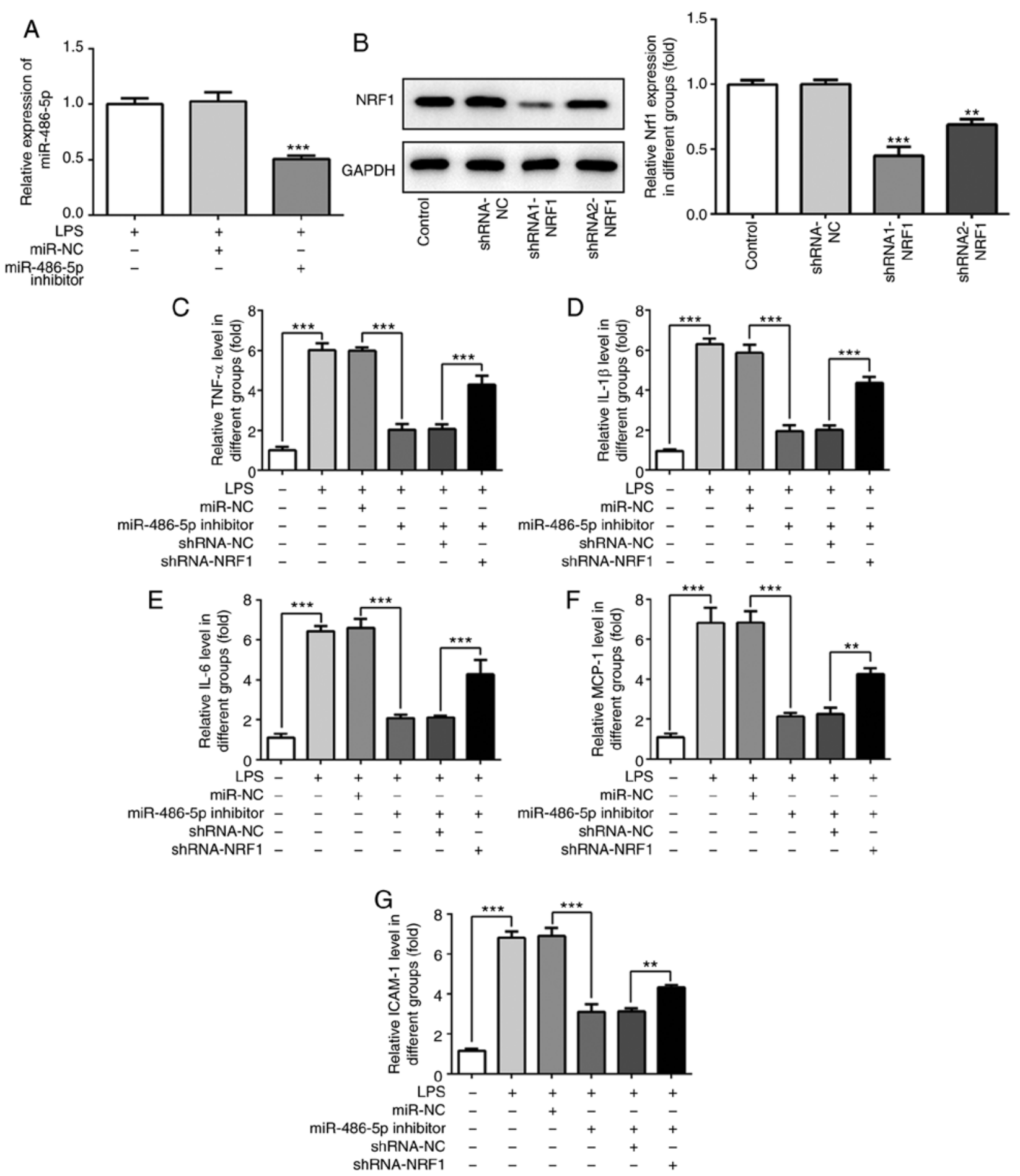

Figure 3. Effect of miR-486-5p on inflammatory response in LPS-induced ATDC5 cells. (A) Following transfection with the miR-486-5p inhibitor, the mRNA level of miR-486-5p was detected using reverse transcription-quantitative PCR. ${ }^{* * * *} \mathrm{P}<0.001$ vs. LPS and miR-NC groups. (B) Following transfection with the shRNA-NRF1, the protein expression of NRF1 was detected using western blotting. ${ }^{* *} \mathrm{P}<0.01$ and ${ }^{* * *} \mathrm{P}<0.001$ vs. control. (C-G) Levels of inflammatory cytokines (TNF- $\alpha$, IL-1 $\beta$, IL-6, MCP-1 and ICAM-1) were detected using ELISA. Data were generated from at least 3 experiments. ${ }^{* *} \mathrm{P}<0.01$ and ${ }^{* * * *} \mathrm{P}<0.001$. miR, microRNA; LPS, lipopolysaccharide; NRF1, nuclear factor erythroid 2 like 1; TNF- $\alpha$, tumor necrosis factor $\alpha$; IL, interleukin; MCP-1, monocyte chemotactic protein 1; ICAM-1, intercellular adhesion molecule 1; shRNA, short hairpin RNA; NC, negative control.

effect of miR-486-5p was decreased by co-transfection with shRNA-NRF1. These results suggested that miR-486-5p could attenuate cell apoptosis in LPS-induced ATDC5 cells by targeting NRF1.

miR-486-5p regulates $L P S$-induced oxidative stress in ADTC5 cells by targeting NRF1. The results presented in
Fig. 5A-E demonstrate the changes in oxidative stress markers in the LPS-induced ATDC5 cells. As shown in Fig. 5A-D, LPS induction significantly increased the activity of ROS and the levels of MDA and LDH, and decreased the activity of SOD. Additionally, antioxidant enzymes such as SOD1, HO-1 and NQO-1 were significantly decreased following treatment with LPS, which is indicative of oxidase and anti-oxidase imbal- 


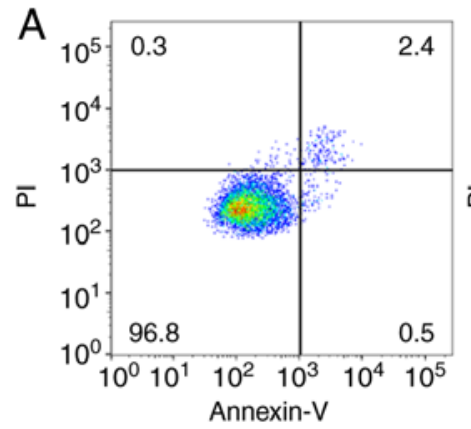

Control

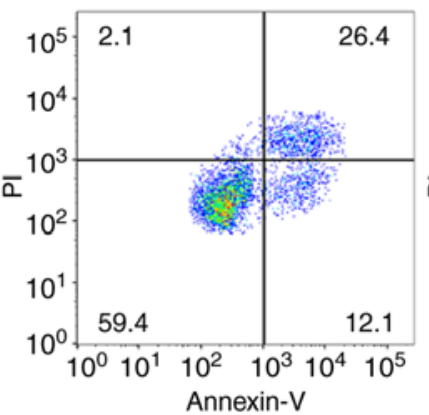

LPS

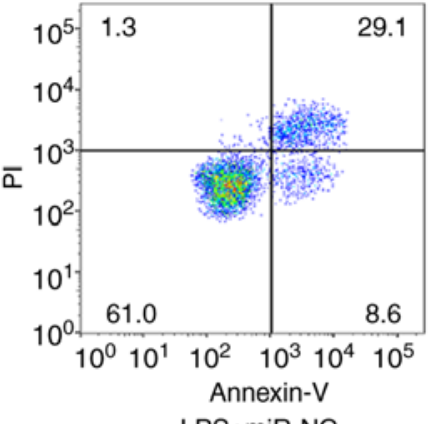

LPS+miR-NC

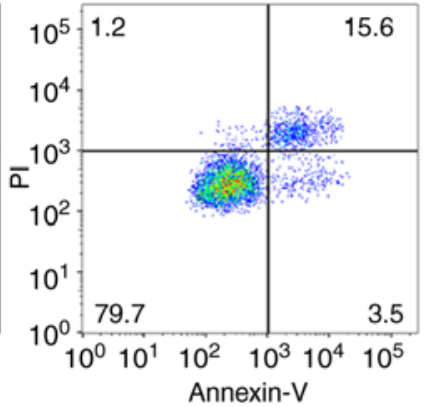

LPS+miR-486-5p inhibitor

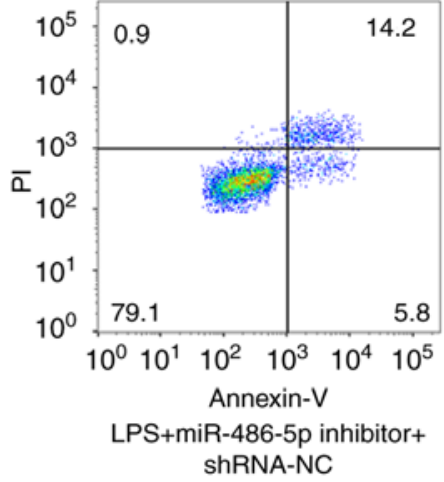

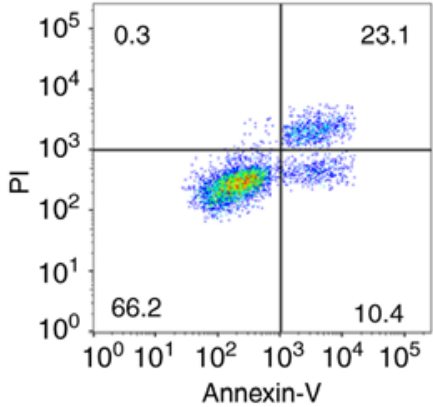

LPS+miR-486-5p inhibitor+ ShRNA-NRF1

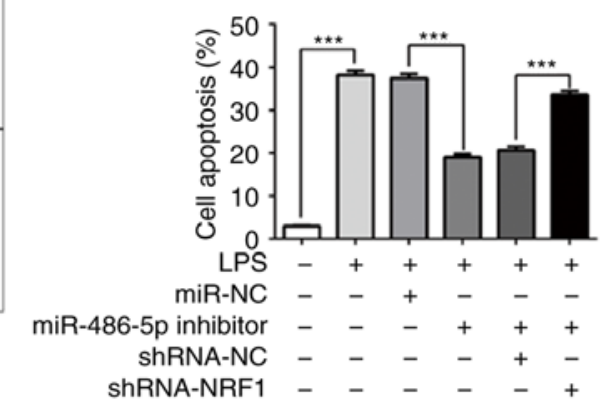

B

B $\quad$ Bax

$\mathrm{Bcl}-2$

Cleaved

Caspase-3

GAPDH

$$
\text { LPS }
$$

miR-NC

miR-486-5p inhibitor

ShRNA-NC ShRNA-NRF

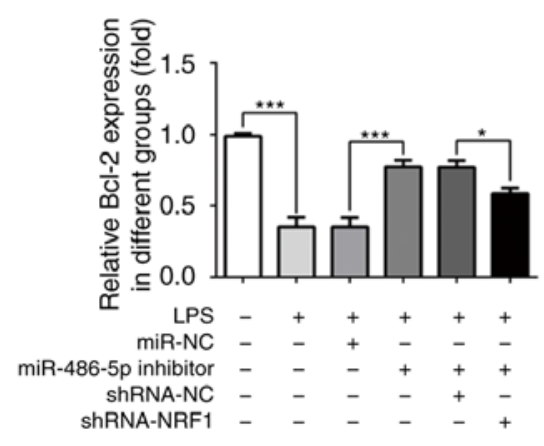

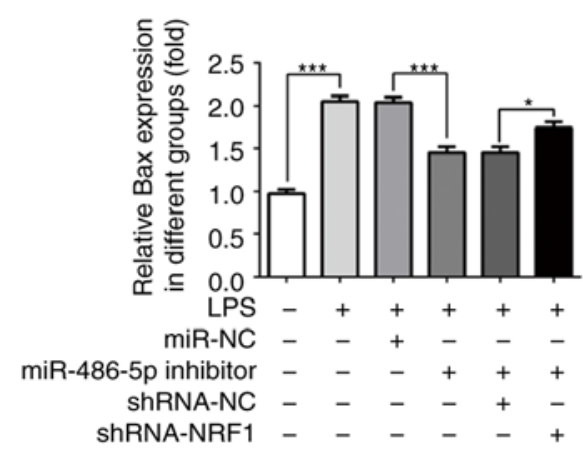

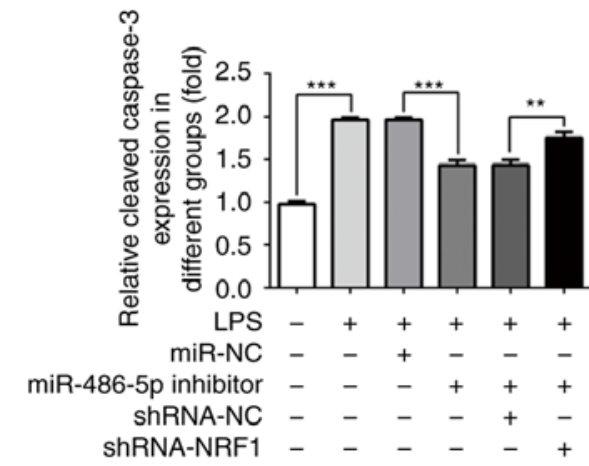

Figure 4. Effect of miR-486-5p on cell apoptosis in LPS-induced ATDC5 cells. (A) Flow cytometry analysis was performed to detect apoptotic rate of ATDC5 cell. (B) Protein expression levels of Bax, Bcl-2, cleaved caspase-3 and caspase-3 were detected using western blotting. Data were generated from at least 3 experiments. ${ }^{*} \mathrm{P}<0.05,{ }^{* *} \mathrm{P}<0.01$ and ${ }^{* * *} \mathrm{P}<0.001$. miR, microRNA; LPS, lipopolysaccharide; NRF1, nuclear factor erythroid 2 like 1; shRNA, short hairpin RNA; NC, negative control.

ance and may result in oxidative stress injury. Inhibition of miR-486-5p significantly decreased the activity of ROS, decreased the levels of MDA and LDH, and increased the activity of SOD, as well as increased the expression of antioxidant enzymes. These data indicated a decrease in oxidative stress following transfection with the miR-486-5p inhibitor. However, co-transfection with shRNA-NRF1 abrogated this effect. These results suggested that inhibition of miR-486-5p could alleviate LPS-induced oxidative stress in ATDC5 cells by targeting NRF1. 

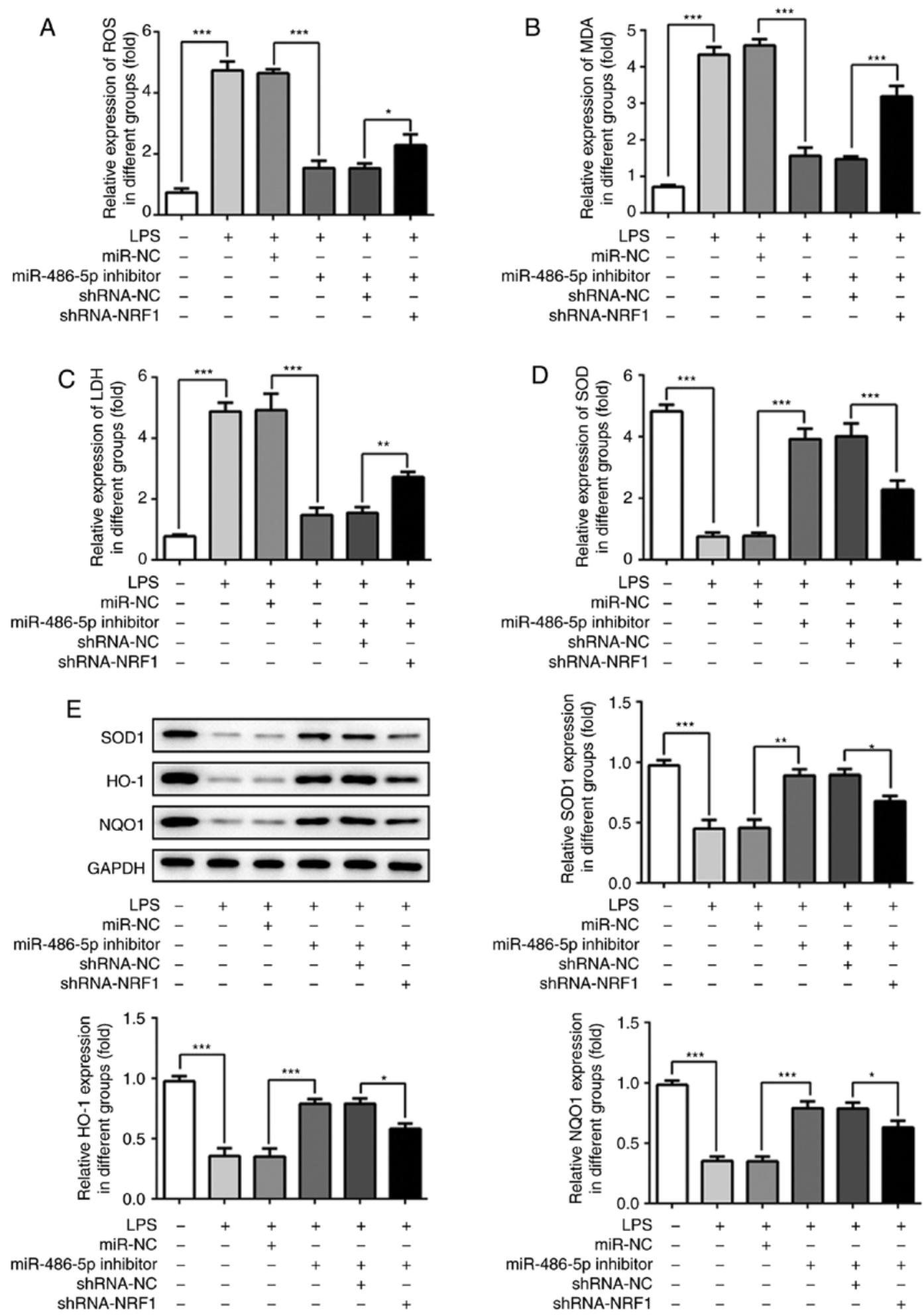

Figure 5. Effect of miR-486-5p on oxidative stress in LPS-induced ATDC5 cells. Levels of (A) ROS, (B) MDA, (C) LDH and (D) SOD were determined using corresponding test kits. (E) Protein expression levels of antioxidant enzymes (SOD1, HO-1 and NQO-1) were detected using western blotting. Data were generated from at least 3 experiments. ${ }^{*} \mathrm{P}<0.0 .5,{ }^{* *} \mathrm{P}<0.01$ and ${ }^{* * * *} \mathrm{P}<0.001$. miR, microRNA; LPS, lipopolysaccharide; NRF1, nuclear factor erythroid 2 like 1; ROS, reactive oxygen species; MDA, malondialdehyde; LDH, lactate dehydrogenase; SOD, superoxide dismutase; HO-1, heme oxygenase 1; NQO-1, NAD(P) $\mathrm{H}$ quinone dehydrogenase 1; NC, negative control; shRNA, short hairpin RNA

\section{Discussion}

The present study revealed that miR-486-5p was upregulated while NRF1 was downregulated in LPS-treated ATDC5 cells. Further investigation demonstrated that NRF1 was a direct target of miR-486-5p, and that miR-486-5p serves an important role in regulating cell damage in LPS-induced ATDC5 cells by targeting NRF1. OA is generally accompanied by oxidative stress, apoptosis and inflammation, which are involved in cartilage damage (15). In the present study, an increased 
production of inflammatory cytokines, severe oxidative stress injury and increased apoptosis were observed in LPS-induced ATDC5 cells. Inhibition of miR-486-5p effectively suppressed inflammation, oxidative stress and apoptosis induced by LPS, whereas the inhibitor effect of downregulated miR-486-5p was decreased by inhibiting NRF1. Additionally, the decreased expressions of SOD1, HO-1 and NQO-1 as a result of LPS treatment was improved by the inhibition of miR-486-5p. Decreasing the expression of NRF1 abrogated this effect. These results indicated that the inhibition of miR-486-5p may have significant therapeutic potential in OA.

The degeneration of articular cartilage is one of the primary causes underlying the development of OA (16). The inflammatory response of articular cartilage accompanied by the release of inflammatory cytokines from chondrocytes is responsible for the degeneration of articular cartilage, and therefore for the occurrence and development of OA $(17,18)$. LPS induced inflammatory injury in various cells by promoting the production and release of inflammatory cytokines, including TNF- $\alpha$, IL-6 and IL-1 $\beta$, which were involved in the inflammatory response of OA $(19,20)$. At present, LPS-induced chondrocytes are widely used as a cell model to simulate the inflammation of articular cartilage in order to investigate the mechanisms underlying $\mathrm{OA}$ and to identify more effective therapeutic targets $(18,21)$. An increase in the release of TNF- $\alpha$, IL-6, IL-1 $\beta$ and MCP-1 was detected in LPS-induced chondrogenic ATDC5 cells in the present study. Inhibition of miR-486-5p decreased the release of inflammatory cytokines, suggesting that the inhibition of miR-486-5p was beneficial and suppressed LPS-induced inflammatory injury in ATDC5 cells. ICAM-1, a type of cell adhesion molecule, is an immunoglobulin-like transmembrane protein that is induced by inflammatory cytokines in endothelial cells (22). Therefore, the expression of ICAM-1 is generally upregulated by inflammatory cytokines such as TNF- $\alpha$ and IL-6 $(23,24)$. The present study revealed that ICAM-1 was upregulated in LPS-induced ATDC5 cells in response to the inflammatory cytokines. The expression of ICAM-1 was downregulated following the inhibition of miR-486-5p, reflecting the decrease in inflammatory injury.

The pathological process of OA is complex and multifactorial, and chondrocyte apoptosis is crucial in the progression of OA (25). Chondrocyte apoptosis has been observed in cell and animal models of OA $(25,26)$. Inhibition of cell apoptosis has been an effective approach to screen for beneficial drugs in the treatment of OA $(25,26)$. The present study revealed that LPS significantly increased the rate of apoptosis, which was then decreased after inhibition of miR-486-5p. In addition, the increased expression of Bcl-2, and decreased expression levels of Bax and cleaved caspase-3 following the inhibition of miR-486-5p indicated that miR-486-5p may exert its function by regulating the expression of apoptosis-related proteins.

Previous studies have revealed that HO- 1 is an inducible enzyme that exerts anti-inflammatory, antioxidant and anti-apoptotic functions $(27,28)$. It has been reported that the activation of HO-1 may protect against oxidative stress induced by ethanol in LO2 cells (29). Inhibition of HO-1 promoted the activation of the NF- $x \mathrm{~B}$ signaling pathway and the production of pro-inflammatory cytokines (30). It was also reported that $\mathrm{HO}-1$ regulated oxidative stress and apoptosis in $\mathrm{OA}$ chondrocytes (31). In the present study, HO-1 was significantly downregulated in chondrocytes induced by LPS-induced ATDC5 cells, and was upregulated following miR-486-5p inhibition. This suggested that the anti-inflammatory, antioxidant and anti-apoptotic effects of miR-486-5p inhibition in LPS-induced ATDC5 cells may be attributed to the downregulation of HO-1. Oxidative stress and generation of free radicals, as primary and secondary events, have contributed to a large number of diseases, including OA (32). The gene of SOD1, NQO-1, as well as ROS, MDA, LDH and total SOD serve an important role in cytoprotection against oxidative stress (33-35). The present study revealed that the anti-oxidative activity of miR-486-5p inhibition was reflected in the increased expression of antioxidant-associated genes such as HO-1, NQO-1 and SOD, and the decreased expression of ROS, MDA and LDH.

The present study revealed the effect of miR-486-5p on the LPS-induced inflammatory response, oxidative stress and apoptosis in ATDC5 cells. Therefore, further investigation was performed to elucidate the target gene and regulatory mechanism of miR-486-5p. The results revealed that NRF1 is a direct target of miR-486-5p. NRF1 is an important regulator involved in cellular oxidative stress and inflammatory injury. In NRF1-knockout mice, the expression levels of HO-1 and NQO-1 genes were significantly increased, indicating that NRF1 knockout alleviates the oxidative stress response $(36,37)$. In addition to mediating the expression of antioxidant genes, NRF1 is also implicated in cellular immune responses and mitochondrial homeostasis (38). In the present study, the effect of miR-496-5p inhibition was diminished following co-transfection with shRNA-NRF1, indicating that miR-496-5p may exert its functions by regulating the expression of NRF1. This result demonstrated that NRF1 participated in the inflammatory response and oxidative stress during the progression of OA.

In conclusion, the present study demonstrated that inhibition of miR-486-5p alleviated LPS-induced cell damage in ATDC5 cells. Furthermore, NFR1 was shown to be a target of miR-486-5p, and inhibition of miR-486-5p mitigated inflammation, oxidative stress and apoptosis by regulating NFR1. The results of the present study may provide a novel viewpoint regarding miR-486-5p as potential therapeutic target for OA.

\section{Acknowledgements}

Not applicable.

\section{Funding}

No funding was received.

\section{Availability of data and materials}

All data generated or analyzed during this study are included in this published article.

\section{Authors' contributions}

QC designed and conceived the experiments. QC, MJ and CL performed the experiments. QC and RG analyzed the data. QC, MJ and CL wrote the manuscript. MJ and RG performed the software analyses. All authors read and approved the final manuscript. 


\section{Ethics approval and consent to participate}

Not applicable.

\section{Patient consent for publication}

Not applicable.

\section{Competing interests}

The authors declare that they have no competing interests.

\section{References}

1. Dieppe PA and Lohmander LS: Pathogenesis and management of pain in osteoarthritis. Lancet 365: 965-973, 2005.

2. Zhou Y, Ming J, Li Y, Du X, Deng M, He B, Zhou J, Wang G and Liu S: Surfactant protein D attenuates nitric oxide-stimulated apoptosis in rat chondrocyte by suppressing p38 MAPK signaling. Biochem Biophys Res Commun 495: 526-532, 2018.

3. Sacitharan PK: Ageing and Osteoarthritis. Subcell Biochem 91 123-159, 2019.

4. Jones G: What's new in osteoarthritis pathogenesis? Intern Med J 46: 229-236, 2016.

5. Yucesoy B, Charles LE, Baker B and Burchfiel CM: Occupational and genetic risk factors for osteoarthritis: A review. Work 50 261-273, 2015

6. Jin H, Zhang H, Ma T, Lan H, Feng S, Zhu H and Ji Y: Resveratrol protects murine chondrogenic ATDC5 cells against LPS-induced inflammatory injury through up-regulating miR-146b. Cell Physiol Biochem 47: 972-980, 2018.

7. Bugno M, Daniel M, Chepelev NL and Willmore WG: Changing gears in Nrf1 research, from mechanisms of regulation to its role in disease and prevention. Biochim Biophys Acta 1849: 1260-1276, 2015

8. Kim J, Xing W, Wergedal J, Chan JY and Mohan S: Targeted disruption of nuclear factor erythroid-derived 2-like 1 in osteoblasts reduces bone size and bone formation in mice. Physio Genomics 40: 100-110, 2010.

9. $\mathrm{Li} \mathrm{G}, \mathrm{Han} \mathrm{N}, \mathrm{Li} \mathrm{Z}$ and $\mathrm{Lu} \mathrm{Q}$ : Identification of transcription regulatory relationships in rheumatoid arthritis and osteoarthritis Clin Rheumatol 32: 609-615, 2013.

10. Park SY, Kim SH, Yoon HK, Yim CH and Lim SK: The role of nuclear factor-E2-related factor 1 in the oxidative stress response in MC3T3-E1 osteoblastic cells. Endocrinol Metab (Seoul) 31: 336-342, 2016.

11. Nugent M: MicroRNAs: Exploring new horizons in osteoarthritis. Osteoarthritis Cartilage 24: 573-580, 2016.

12. Kong R, Gao J, Si Y and Zhao D: Combination of circulating miR-19b-3p, miR-122-5p and miR-486-5p expressions correlates with risk and disease severity of knee osteoarthritis. Am J Trans Res 9: 2852-2864, 2017.

13. Shi J, Guo K, Su S, Li J and Li C: miR-486-5p is upregulated in osteoarthritis and inhibits chondrocyte proliferation and migration by suppressing SMAD2. Mol Med Rep 18: 502-508, 2018.

14. Livak KJST and Schmittgen TD: Analysis of relative gene expression data using real-time quantitative PCR and the 2(-Delta Delta C(T)) Method. Methods 25: 402-408, 2001.

15. Mongkhon JM, Thach M, Shi Q, Fernandes JC, Fahmi H and Benderdour M: Sorbitol-modified hyaluronic acid reduces oxidative stress, apoptosis and mediators of inflammation and catabolism in human osteoarthritic chondrocytes. Inflamm Res 63: 691-701, 2014.

16. Murata K, Kanemura N, Kokubun T, Fujino T, Morishita Y, Onitsuka K, Fujiwara S, Nakajima A, Shimizu D and Takayanagi K: Controlling joint instability delays the degeneration of articular cartilage in a rat model. Osteoarthritis Cartilage 25: 297-308, 2017.

17. Saklatvala J: Inflammatory signaling in cartilage: MAPK and NF-kappaB pathways in chondrocytes and the use of inhibitors for research into pathogenesis and therapy of osteoarthritis. Curr Drug Targets 8: 305-313, 2007.

18. Pan L, Liu D, Zhao L, Wang L, Xin M and Li X: Long noncoding RNA MALAT1 alleviates lipopolysaccharide-induced inflammatory injury by upregulating microRNA-19b in murine chondrogenic ATDC5 cells. J Cell Biochem 119: 10165-10175, 2018.
19. Wu DP, Zhang JL, Wang JY, Cui MX, Jia JL, Liu XH and Liang QD: MiR-1246 promotes LPS-induced inflammatory injury in chondrogenic cells ATDC5 by targeting HNF4 $\gamma$. Cell Physiol Biochem 43: 2010-2021, 2017.

20. Scotece M, Conde J, Abella V, López V, Francisco V, Ruiz C, Campos V, Lago F, Gomez R, Pino J, et al: Oleocanthal inhibits catabolic and inflammatory mediators in LPS-activated human primary osteoarthritis (OA) chondrocytes through MAPKs/NF- $x$ B pathways. Cell Physiol Biochem 49: 2414-2426, 2018.

21. Wang Y and Kong D: MicroRNA-136 promotes lipopolysaccharide-induced ATDC5 cell injury and inflammatory cytokine expression by targeting myeloid cell leukemia 1. J Cell Biochem 119: 9316-9326, 2018.

22. Hubbard AK and Rothlein R: Intercellular adhesion molecule-1 (ICAM-1) expression and cell signaling cascades. Free Radic Biol Med 28: 1379-1386, 2000.

23. Dadgar Pakdel F, Keramatipour M, Noorbakhsh F, Talebi S and Vodjgani M: Investigating the effect of rs3783605 single-nucleotide polymorphism on the activity of VCAM-1 promoter in human umbilical vein endohelial cells. Iran J Allergy Asthma Immunol 14: 179-187, 2015.

24. Yu GI, Jun SE and Shin DH: Associations of VCAM-1 gene polymorphisms with obesity and inflammation markers. Inflamm Res 66: 217-225, 2017

25. Liang $Z$ and Ren C: Emodin attenuates apoptosis and inflammation induced by LPS through up-regulating lncRNA TUG1 in murine chondrogenic ATDC5 cells. Biomed Pharmacother 103: 897-902, 2018

26. Pan T, Shi X, Chen H, Chen R, Wu D, Lin Z, Zhang J and Pan J: Geniposide suppresses interleukin-1 $\beta$-induced inflammation and apoptosis in rat chondrocytes via the PI3K/Akt/NF- $x \mathrm{~B}$ signaling pathway. Inflammation 41: 390-399, 2018

27. Lu DY, Chen JH, Tan TW, Huang CY, Yeh WL and Hsu HC: Resistin protects against 6-hydroxydopamine-induced cell death in dopaminergic-like MES23.5 cells. J Cell Physiol 228: 563-571, 2013.

28. Xiong J, Wang K, Yuan C, Xing R, Ni J, Hu G, Chen F and Wang X: Luteolin protects mice from severe acute pancreatitis by exerting $\mathrm{HO}-1$-mediated anti-inflammatory and antioxidant effects. Int J Mol Med 39: 113-125, 2017.

29. Zeng T, Zhang CL, Song FY, Zhao XL, Yu LH, Zhu ZP and Xie KQ: The activation of $\mathrm{HO}-1 / \mathrm{Nrf}-2$ contributes to the protective effects of diallyl disulfide (DADS) against ethanol-induced oxidative stress. Biochim Biophys Acta 1830: 4848-4859, 2013

30. Luo D, Guo Y, Cheng Y, Zhao J, Wang Y and Rong J: Natural product celastrol suppressed macrophage M1 polarization against inflammation in diet-induced obese mice via regulating Nrf2/HO-1, MAP kinase and NF- $x$ B pathways. Aging (Albany NY) 9: 2069-2082, 2017.

31. Sun J, Wei X, Lu Y, Cui M, Li F, Lu J, Liu Y and Zhang X: Glutaredoxin 1 (GRX1) inhibits oxidative stress and apoptosis of chondrocytes by regulating $\mathrm{CREB} / \mathrm{HO}-1$ in osteoarthritis. Mol Immunol 90: 211-218, 2017

32. Lepetsos $P$ and Papavassiliou AG: ROS/oxidative stress signaling in osteoarthritis. Biochim Biophys Acta 1862: 576-591, 2016.

33. Zhang Y, Unnikrishnan A, Deepa SS, Liu Y, Li Y, Ikeno Y, Sosnowska D, Van Remmen $\mathrm{H}$ and Richardson A: A new role for oxidative stress in aging: The accelerated aging phenotype in Sod1-/- mice is correlated to increased cellular senescence. Redox Biol 11: 30-37, 2017.

34. Lv D, Zhou Q, Xia Y, You X, Zhao Z, Li Y and Zou H: The association between oxidative stress alleviation via sulforaphane-induced Nrf2-HO-1/NQO-1 signaling pathway activation and chronic renal allograft dysfunction improvement. Kidney Blood Press Res 43: 191-205, 2018.

35. Samarghandian S, Azimi-Nezhad M, Farkhondeh $T$ and Samini F: Anti-oxidative effects of curcumin on immobilization-induced oxidative stress in rat brain, liver and kidney. Biomed Pharmacother 87: 223-229, 2017.

36. Ohtsuji M, Katsuoka F, Kobayashi A, Aburatani H, Hayes JD and Yamamoto M: Nrf1 and Nrf2 play distinct roles in activation of antioxidant response element-dependent genes. J Biol Chem 283 33554-33562, 2008.

37. Biswas M and Chan JY: Role of Nrf1 in antioxidant response element-mediated gene expression and beyond. Toxicol Appl Pharmacol 244: 16-20, 2010.

38. Kim HM, Han JW and Chan JY: Nuclear factor erythroid-2 Like 1 (NFE2L1): Structure, function and regulation. Gene 584: 17-25, 2016 